\title{
A Crank-Nicolson Difference Scheme for Solving a Type of Variable Coefficient Delay Partial Differential Equations
}

\author{
Wei Gu' and Peng Wang ${ }^{2}$ \\ ${ }^{1}$ School of Statistics and Mathematics, Zhongnan University of Economics and Law, \\ Wuhan 430073, China \\ ${ }^{2}$ School of Mathematics, Jilin University, Changchun 130012, China
}

Correspondence should be addressed to Wei Gu; wei_gu@znufe.edu.cn

Received 24 November 2013; Accepted 23 January 2014; Published 27 February 2014

Academic Editor: Francisco J. Marcellán

Copyright (C) 2014 W. Gu and P. Wang. This is an open access article distributed under the Creative Commons Attribution License, which permits unrestricted use, distribution, and reproduction in any medium, provided the original work is properly cited.

\begin{abstract}
A linearized Crank-Nicolson difference scheme is constructed to solve a type of variable coefficient delay partial differential equations. The difference scheme is proved to be unconditionally stable and convergent, where the convergence order is two in both space and time. A numerical test is provided to illustrate the theoretical results.
\end{abstract}

\section{Introduction}

In the past few years, many scholars pay their attention to the theory of delay differential equations (DDEs) [1, 2]. There are many research results on delay ordinary differential equations [3, 4]; however, only few scholars focus on studies of delay partial differential equations. As we know, since, in most cases, DDEs' exact solutions cannot be computed analytically, efficient numerical methods are needed to solve such equations.

In this paper, the numerical solutions of the following variable coefficient delay partial differential equations are considered:

$$
\begin{gathered}
r(x, t) u_{t}-d u_{x x}=f(u(x, t-s)), \quad(x, t) \in(0,1) \times(0, T], \\
u(x, t)=\phi(x, t), \quad x \in[0,1], t \in[-s, 0], \\
u(0, t)=\alpha(t), \quad u(1, t)=\beta(t), \quad t \in(0, T],
\end{gathered}
$$

where $d>0$ is the constant diffusion coefficient, $s>0$ is the delay term, $\Omega=[0,1] \times[-s, T], r(x, t) \in C((0,1) \times(0, T])$, and $r(x, t) \geq c_{0}>0$. In the case of $r(x, t)=1$, numerical solutions of (1)-(3) have been considered in [5-7]. A CrankNicolson scheme and a linearized compact difference scheme have been proposed by Zhang and Sun in [5] and [6], respectively. Q. F. Zhang and C. J. Zhang considered a new linearized compact multisplitting scheme in [7]. We will construct a Crank-Nicolson scheme for solving (1)-(3). The unconditional stability and convergence will be shown in this paper, where the convergence order is two in both space and time. To testify the theoretical results, a numerical test is provided.

The paper is organized as follows. In Section 2, a linearized Crank-Nicolson scheme is constructed to solve (1)-(3). Section 3 considers the solvability, stability, and convergence of the Crank-Nicolson scheme. In Section 4, a numerical test is provided to illustrate the theoretical results. Section 5 gives a brief discussion of this paper.

\section{Construction of the Linearized Crank-Nicolson Scheme}

In this subsection, a linearized Crank-Nicolson scheme for solving (1)-(3) is constructed. In this paper, we make the following assumptions:

(H1) assume that (1)-(3) had a unique solution $u \in$ $C^{4,3}(\Omega), u$, and its partial derivatives are bounded by a constant $c_{1}$; 
(H2) $f(u(x, t-s))$ has second derivatives, and we denote

$$
\begin{aligned}
& c_{2}=\max _{\left|\varepsilon_{1}\right| \leq \varepsilon_{0},\left|\varepsilon_{2}\right| \leq \varepsilon_{0},\left|\varepsilon_{3}\right| \leq \varepsilon_{0}}\left\{\left|f\left(u(x, t-s)+\varepsilon_{1}\right)\right|,\right. \\
&\left|f^{\prime}\left(u(x, t-s)+\varepsilon_{2}\right)\right|, \\
&\left.\left|f^{\prime \prime}\left(u(x, t-s)+\varepsilon_{3}\right)\right|\right\},
\end{aligned}
$$

where $\varepsilon_{0}>0$, and $c_{2}$ are constants.

Two positive integers $M$ and $j$ are taken; then let $h=$ $1 / M, \tau=s / j, x_{i}=i h, t_{k}=k \tau$ and $t_{k+1 / 2}=\left(t_{k}+t_{k+1}\right) / 2$. Define $\Omega_{h \tau}=\Omega_{h} \times \Omega_{\tau}$, where $\Omega_{h}=\left\{x_{i} \mid 0 \leq i \leq M\right\}$ and $\Omega_{\tau}=\left\{t_{k} \mid-j \leq k \leq N\right\}, N=[T / \tau]$. Denote $U_{i}^{k}=u\left(x_{i}, t_{k}\right)$, $0 \leq i \leq M,-j \leq k \leq N$. Let

$$
\mathscr{W}=\left\{v_{i}^{k} \mid 0 \leq i \leq M,-j \leq k \leq N\right\}
$$

be the grid function space defined on $\Omega_{h \tau}$. Introduce the following notations:

$$
\begin{array}{cc}
v_{i}^{k+1 / 2}=\frac{v_{i}^{k}+v_{i}^{k+1}}{2}, & \delta_{t} v_{i}^{k+1 / 2}=\frac{v_{i}^{k+1}-v_{i}^{k}}{\tau}, \\
\delta_{x} v_{i+1 / 2}^{k}=\frac{v_{i+1}^{k}-v_{i}^{k}}{h}, & \delta_{x}^{2} v_{i}^{k}=\frac{v_{i+1}^{k}-2 v_{i}^{k}+v_{i-1}^{k}}{h^{2}} .
\end{array}
$$

Considering (1) at the point $\left(x_{i}, t_{k+1 / 2}\right)$, we have

$$
\begin{aligned}
& r\left(x_{i}, t_{k+1 / 2}\right) \frac{\partial u}{\partial t}\left(x_{i}, t_{k+1 / 2}\right)-d \frac{\partial^{2} u}{\partial x^{2}}\left(x_{i}, t_{k+1 / 2}\right) \\
& \quad=f\left(u\left(x_{i}, t_{k+(1 / 2)-j}\right)\right), \quad 1 \leq i \leq M-1,0 \leq k \leq N-1 .
\end{aligned}
$$

From Taylor expansion,

$$
\begin{aligned}
& \frac{\partial u}{\partial t}\left(x_{i}, t_{k+1 / 2}\right)=\delta_{t} U_{i}^{k+1 / 2}-\frac{\tau^{2}}{24} \frac{\partial^{3} u}{\partial t^{3}}\left(x_{i}, \eta_{i}^{k}\right), \eta_{i}^{k} \in\left(t_{k}, t_{k+1}\right), \\
& \frac{\partial^{2} u}{\partial x^{2}}\left(x_{i}, t_{k+1 / 2}\right) \\
&=\frac{1}{2}\left[\frac{\partial^{2} u}{\partial x^{2}}\left(x_{i}, t_{k}\right)+\frac{\partial^{2} u}{\partial x^{2}}\left(x_{i}, t_{k+1}\right)\right] \\
&-\frac{\tau^{2}}{8} \frac{\partial^{4} u}{\partial x^{2} \partial t^{2}}\left(x_{i}, \gamma_{i}^{k}\right) \\
&=\frac{1}{2}\left(\delta_{x}^{2} U_{i}^{k}+\delta_{x}^{2} U_{i}^{k+1}\right) \\
&-\frac{h^{2}}{24}\left[\frac{\partial^{4} u}{\partial x^{4}}\left(\xi_{i}^{k}, t_{k}\right)+\frac{\partial^{4} u}{\partial x^{4}}\left(\xi_{i}^{k+1}, t_{k+1}\right)\right] \\
&-\frac{\tau^{2}}{8} \frac{\partial^{4} u}{\partial x^{2} \partial t^{2}}\left(x_{i}, \gamma_{i}^{k}\right), \\
& \xi_{i}^{k}, \xi_{i}^{k+1} \in\left(x_{i-1}, x_{i+1}\right), \\
& \gamma_{i}^{k} \in\left(t_{k}, t_{k+1}\right),
\end{aligned}
$$

$$
\begin{aligned}
f( & \left.u\left(x_{i}, t_{k+(1 / 2)-j}\right)\right) \\
= & f\left(u\left(x_{i}, t_{k-j}\right)\right) \\
& +\frac{\tau}{2} f^{\prime}\left(u\left(x_{i}, t_{k-j}\right)\right) u_{t}^{\prime}\left(x_{i}, t_{k-j}\right)+\zeta_{i}^{k-j} \\
= & f\left(U_{i}^{k-j}\right)+\frac{\tau}{2} f^{\prime}\left(U_{i}^{k-j}\right) \delta_{t} U_{i}^{k+(1 / 2)-j} \\
& +\zeta_{i}^{k-j}, \quad \zeta_{i}^{k-j} \in\left(t_{k-j}, t_{k+1-j}\right),
\end{aligned}
$$

where $\left|\zeta_{i}^{k-j}\right| \leq c_{3} \tau^{2}$. Substituting (8) into (7) and denoting $r_{i}^{k+1 / 2}=r\left(x_{i}, t_{k+1 / 2}\right)$, we obtain

$$
\begin{aligned}
r_{i}^{k+1 / 2} & \delta_{t} U_{i}^{k+1 / 2}-d \delta_{x}^{2} U_{i}^{k+1 / 2} \\
& =f\left(U_{i}^{k-j}\right)+\frac{\tau}{2} f^{\prime}\left(U_{i}^{k-j}\right) \delta_{t} U_{i}^{k+(1 / 2)-j}+R_{i}^{k},
\end{aligned}
$$

where

$$
\left|R_{i}^{k}\right| \leq c_{4}\left(\tau^{2}+h^{2}\right), \quad 1 \leq i \leq M-1,0 \leq k \leq N-1 .
$$

Discretizing the initial and boundary conditions of (2) and (3), we obtain

$$
\begin{aligned}
& U_{i}^{k}=\phi\left(x_{i}, t_{k}\right), \quad 0 \leq i \leq M,-j \leq k \leq 0, \\
& U_{0}^{k}=\alpha\left(t_{k}\right), \quad U_{M}^{k}=\beta\left(t_{k}\right), \quad 1 \leq k \leq N .
\end{aligned}
$$

Replacing $U_{i}^{k}$ by $u_{i}^{k}$ and omitting $R_{i}^{k}$, we obtain the following Crank-Nicolson scheme:

$$
\begin{gathered}
r_{i}^{k+1 / 2} \delta_{t} u_{i}^{k+1 / 2}-d \delta_{x}^{2} u_{i}^{k+1 / 2} \\
=f\left(u_{i}^{k-j}\right)+\frac{\tau}{2} f^{\prime}\left(u_{i}^{k-j}\right) \delta_{t} u_{i}^{k+(1 / 2)-j}, \\
u_{i}^{k}=\phi\left(x_{i}, t_{k}\right), \quad 0 \leq i \leq M, \quad-j \leq k \leq 0, \\
u_{0}^{k}=\alpha\left(t_{k}\right), \quad u_{M}^{k}=\beta\left(t_{k}\right), \quad 1 \leq k \leq N .
\end{gathered}
$$

\section{The Solvability, Convergence, and Stability of the Crank-Nicolson Scheme}

Define the following grid function space on $\Omega_{h}$ :

$$
V=v \mid v=\left(v_{0}, v_{1}, \ldots, v_{M}\right), \quad v_{0}=v_{M}=0 .
$$

If $v \in V$, introducing the following notations:

$$
\begin{gathered}
\|v\|=\sqrt{h \sum_{i=1}^{M-1}\left(v_{i}\right)^{2}}, \quad|v|_{1}=\sqrt{h \sum_{i=1}^{M}\left(\frac{v_{i}-v_{i-1}}{h}\right)^{2}}, \\
\|v\|_{\infty}=\max _{0 \leq i \leq M}\left|v_{i}\right| .
\end{gathered}
$$

The following two inequalities are satisfied [8]:

$$
\begin{aligned}
& \|v\|_{\infty} \leq \frac{1}{2}|v|_{1}, \\
& \|v\| \leq \frac{1}{\sqrt{6}}|v|_{1} .
\end{aligned}
$$


For the analysis of the difference scheme, the following Lemma is needed.

Lemma 1 (see [8]). Let $\left\{F^{k} \mid k \geq 0\right\}$ be nonnegative sequence and satisfy

$$
F^{k+1} \leq A+B \tau \sum_{i=1}^{k} F^{l}, \quad k=0,1, \ldots
$$

then

$$
F^{k+1} \leq A \exp (B K \tau), \quad k=0,1,2, \ldots,
$$

where $A$ and $B$ are nonnegative constants.

Theorem 2. The difference scheme (13) has a unique solution, under the condition that $h$ and $\tau$ are small enough.

Proof. From the positive definiteness of the coefficient matrix of the scheme (13), we can easily obtain the results of Theorem 2 by the mathematical induction method.

Denoting $e_{i}^{k}=U_{i}^{k}-u_{i}^{k}, 0 \leq i \leq M,-j \leq k \leq N$, subtracting (13) from (9), (11), and (12), respectively, we obtain the following error equations:

$$
\begin{aligned}
& r_{i}^{k+1 / 2} \delta_{t} e_{i}^{k+1 / 2}-d \delta_{x}^{2} e_{i}^{k+1 / 2} \\
& =f\left(U_{i}^{k-j}\right)-f\left(u_{i}^{k-j}\right) \\
& +\frac{\tau}{2}\left[f^{\prime}\left(U_{i}^{k-j}\right) \delta_{t} U_{i}^{k+(1 / 2)-j}\right. \\
& \left.\quad-f^{\prime}\left(u_{i}^{k-j}\right) \delta_{t} u_{i}^{k+(1 / 2)-j}\right]+R_{i}^{k}, \\
& e_{i}^{k}=0, \quad 0 \leq i \leq M,-j \leq k \leq 0, \\
& e_{0}^{k}=0, \quad e_{M}^{k}=0, \quad 1 \leq k \leq N .
\end{aligned}
$$

Theorem 3. Letting $h$ and $\tau$ be small enough, one has

$$
\left\|e^{k}\right\|_{\infty} \leq C\left(\tau^{2}+h^{2}\right), \quad 0 \leq k \leq N,
$$

where $C>0$ is independent of $h$ and $\tau$.

Proof. Multiplying (20) by $h \delta_{t} e_{i}^{k+1 / 2}$ and summing up for $i$ from 1 to $M-1$, we obtain

$$
h \sum_{i=1}^{M-1} r_{i}^{k+1 / 2}\left(\delta_{t} e_{i}^{k+1 / 2}\right)^{2}-d h \sum_{i=1}^{M-1} \delta_{x}^{2} e_{i}^{k+1 / 2} \delta_{t} e_{i}^{k+1 / 2}=I_{1}+I_{2},
$$

where

$$
\begin{gathered}
I_{1}=h \sum_{i=1}^{M-1}\left\{f\left(U_{i}^{k-j}\right)-f\left(u_{i}^{k-j}\right)\right. \\
+\frac{\tau}{2}\left[f^{\prime}\left(U_{i}^{k-j}\right) \delta_{t} U_{i}^{k+(1 / 2)-j}\right. \\
\left.\left.-f^{\prime}\left(u_{i}^{k-j}\right) \delta_{t} u_{i}^{k+(1 / 2)-j}\right]\right\} \delta_{t} e_{i}^{k+1 / 2}, \\
I_{2}=h \sum_{i=1}^{M-1} R_{i}^{k} \delta_{t} e_{i}^{k+1 / 2} .
\end{gathered}
$$

The mathematical induction method will be used to prove Theorem 3. From (21), we have $\left\|e^{k}\right\|_{\infty}=0$, for $-j \leq k \leq 0$. Suppose that (23) is true for $0<k \leq l$, we will prove that (23) is also valid for $k=l+1$.

From the inductive assumption, we have

$$
\left\|e^{k}\right\|_{\infty} \leq C\left(\tau^{2}+h^{2}\right), \quad 0 \leq k \leq l .
$$

In the following, each term of (24) will be estimated. Consider

$$
\begin{gathered}
h \sum_{i=1}^{M-1} r_{i}^{k+1 / 2}\left(\delta_{t} e_{i}^{k+1 / 2}\right)^{2} \geq c_{0}\left\|\delta_{t} e^{k+1 / 2}\right\|^{2}, \\
-d h \sum_{i=1}^{M-1} \delta_{x}^{2} e_{i}^{k+1 / 2} \delta_{t} e_{i}^{k+1 / 2}=\frac{d}{2 \tau}\left(\left|e^{k+1}\right|_{1}^{2}-\left|e^{k}\right|_{1}^{2}\right) .
\end{gathered}
$$

From (H1) and (H2), we have

$$
\begin{gathered}
f\left(U_{i}^{k-j}\right)-f\left(u_{i}^{k-j}\right)+\frac{\tau}{2}\left[f^{\prime}\left(U_{i}^{k-j}\right) \delta_{t} U_{i}^{k+(1 / 2)-j}\right. \\
\left.-f^{\prime}\left(u_{i}^{k-j}\right) \delta_{t} u_{i}^{k+(1 / 2)-j}\right] \\
\leq c_{2}\left|e_{i}^{k-j}\right|+\frac{\tau}{2}\left[f^{\prime}\left(U_{i}^{k-j}\right) \delta_{t} e_{i}^{k+(1 / 2)-j}\right. \\
+\left(f^{\prime}\left(U_{i}^{k-j}\right)-f^{\prime}\left(u_{i}^{k-j}\right)\right) \\
\left.\times\left(\delta_{t} U_{i}^{k+(1 / 2)-j}-\delta_{t} e_{i}^{k+(1 / 2)-j}\right)\right] \\
\leq C\left(\left|e_{i}^{k-j}\right|+\left|e_{i}^{k+1-j}\right|\right) .
\end{gathered}
$$

Using the above inequality, we have

$$
\begin{aligned}
I_{1} & \leq C h \sum_{i=1}^{M-1}\left(\left|e_{i}^{k-j}\right|+\left|e_{i}^{k+1-j}\right|\right)\left|\delta_{t} e_{i}^{k+1 / 2}\right| \\
& \leq \varepsilon h \sum_{i=1}^{M-1}\left(\delta_{t} e_{i}^{k+1 / 2}\right)^{2}+C h\left(\sum_{i=1}^{M-1}\left(e_{i}^{k-j}\right)^{2}+\sum_{i=1}^{M-1}\left(e_{i}^{k+1-j}\right)^{2}\right) \\
& =\varepsilon\left\|\delta_{t} e^{k+1 / 2}\right\|^{2}+C\left(\left\|e^{k-j}\right\|^{2}+\left\|e^{k+1-j}\right\|^{2}\right), \\
I_{2} & \leq \varepsilon h \sum_{i=1}^{M-1}\left(\delta_{t} e_{i}^{k+1 / 2}\right)^{2}+C h \sum_{i=1}^{M-1}\left(R_{i}^{k}\right)^{2} \\
& \leq \varepsilon\left\|\delta_{t} e^{k+1 / 2}\right\|^{2}+C\left(\tau^{2}+h^{2}\right)^{2} .
\end{aligned}
$$

Inserting (27)-(29) into (24), we obtain

$$
\begin{aligned}
c_{0}\left\|\delta_{t} e^{k+1 / 2}\right\|^{2}+\frac{d}{2 \tau}\left(\left|e^{k+1}\right|_{1}^{2}-\left|e^{k}\right|_{1}^{2}\right) \\
\leq 2 \varepsilon\left\|\delta_{t} e^{k+1 / 2}\right\|^{2}+C\left(\left\|e^{k-j}\right\|^{2}+\left\|e^{k+1-j}\right\|^{2}\right) \\
+C\left(\tau^{2}+h^{2}\right)^{2}, \quad 0 \leq k \leq l .
\end{aligned}
$$


Taking $\varepsilon=c_{0} / 2$, we have

$$
\begin{aligned}
\frac{d}{2 \tau}\left(\left|e^{k+1}\right|_{1}^{2}-\left|e^{k}\right|_{1}^{2}\right) \leq & C\left(\left\|e^{k-j}\right\|^{2}+\left\|e^{k+1-j}\right\|^{2}\right) \\
& +C\left(\tau^{2}+h^{2}\right)^{2}, \quad 0 \leq k \leq l
\end{aligned}
$$

The above inequality has the following form:

$$
\begin{gathered}
\left|e^{k+1}\right|_{1}^{2} \leq\left|e^{k}\right|_{1}^{2}+C \tau\left(\left\|e^{k-j}\right\|^{2}+\left\|e^{k+1-j}\right\|^{2}\right) \\
+C \tau\left(\tau^{2}+h^{2}\right)^{2}, \quad 0 \leq k \leq l .
\end{gathered}
$$

Summing up (32) for $k$, noticing (21), and exploiting (17), we have

$$
\begin{aligned}
\left|e^{k+1}\right|_{1}^{2} \leq & \left|e^{0}\right|_{1}^{2}+C \tau \sum_{m=0}^{k}\left(\left\|e^{m-j}\right\|^{2}+\left\|e^{m+1-j}\right\|^{2}\right) \\
& +C \tau \sum_{k=0}^{l}\left(\tau^{2}+h^{2}\right)^{2} \\
\leq & C \tau \sum_{m=1}^{k+1-j}\left\|e^{m}\right\|^{2}+C\left(\tau^{2}+h^{2}\right)^{2} \\
\leq & C \tau \sum_{m=1}^{k}\left|e^{m}\right|_{1}^{2}+C\left(\tau^{2}+h^{2}\right)^{2}, \quad 0 \leq k \leq l .
\end{aligned}
$$

By Lemma 1, we have

$$
\left|e^{l+1}\right|_{1}^{2} \leq C\left(\tau^{2}+h^{2}\right)^{2}
$$

where $C$ is a constant which depends on $c_{0}, c_{1}, c_{2}, d$, and $T$. From (16), we obtain

$$
\left\|e^{l+1}\right\|_{\infty} \leq C\left(\tau^{2}+h^{2}\right)
$$

By the inductive principle, this completes the proof.

Remark 4. Theorem 3 shows that the convergence order of the variable coefficient delay partial differential equations (1) is $o\left(t^{2}+h^{2}\right)$. However, for the constant coefficient delay partial differential equations $(r(x, t)=1$ in (1)), a Crank-Nicolson scheme with $o\left(t^{2}+h^{2}\right)$ convergence is constructed in [5], and a new difference scheme with $o\left(t^{2}+h^{4}\right)$ convergence is constructed in [9].

To discuss the stability of the difference scheme (13), we consider the following problem:

$$
\begin{gathered}
r(x, t) v_{t}-d v_{x x}=f(v(x, t-s)), \quad(x, t) \in(0,1) \times(0, T], \\
v(x, t)=\phi(x, t)+\psi(x, t), \quad x \in[0,1], t \in[-s, 0], \\
v(0, t)=\alpha(t), \quad v(1, t)=\beta(t), \quad t \in(0, T] .
\end{gathered}
$$

The following difference scheme solving for (36) can be obtained:

$$
\begin{gathered}
r_{i}^{k+1 / 2} \delta_{t} v_{i}^{k+1 / 2}-d \delta_{x}^{2} v_{i}^{k+1 / 2} \\
=f\left(v_{i}^{k-j}\right)+\frac{\tau}{2} f^{\prime}\left(v_{i}^{k-j}\right) \delta_{t} v_{i}^{k+(1 / 2)-j}, \\
v_{i}^{k}=\phi\left(x_{i}, t_{k}\right)+\psi_{i}^{k}, \quad 0 \leq i \leq M,-j \leq k \leq 0, \\
v_{0}^{k}=\alpha\left(t_{k}\right), \quad v_{M}^{k}=\beta\left(t_{k}\right), \quad 1 \leq k \leq N,
\end{gathered}
$$

where $\psi_{i}^{k}$ is a perturbation of $\phi\left(x_{i}, t_{k}\right)$.

Similar to the proof of Theorem 3, the following stability result can be obtained.

Theorem 5. Denote

$$
\eta_{i}^{k}=v_{i}^{k}-u_{i}^{k}, \quad 0 \leq i \leq M,-j \leq k \leq N .
$$

Then, there exist constants $c_{5}$ and $c_{6}$ such that

$$
\left\|\eta^{k}\right\|_{\infty} \leq c_{5} \sqrt{\tau h \sum_{m=-j}^{0} \sum_{i=1}^{M-1}\left(\psi_{i}^{k}\right)^{2}}
$$

under the condition that $h$ and $\tau$ are small enough and $\max _{-j \leq k \leq 0,0 \leq i \leq M}\left|\psi_{i}^{k}\right| \leq c_{6}$.

Remark 6. Under the condition of assumptions (H1) and (H2) and $\max _{-j \leq k \leq 0,0 \leq i \leq M}\left|\psi_{i}^{k}\right| \leq c_{6}$, for small $h$ and $\tau$, we can get the stability results of Theorem 5 (which can be referred to in $[8,10,11])$, where the difficulty is that $r_{i}^{k+1 / 2} \neq 1$; the proof can be referred to in the proof of Theorem 3 .

\section{Numerical Test}

In this section, a numerical example is considered to validate the algorithm provided in this paper, and the numerical solutions $u_{i}^{k}$ of the example are obtained by exploiting scheme (13). Define

$$
E_{\infty}(h, \tau)=\max _{0 \leq i \leq M, 0 \leq k \leq N}\left|u\left(x_{i}, t_{k}\right)-u_{i}^{k}\right| .
$$

Consider the following problem:

$$
\begin{gathered}
r(x, t) u_{t}-u_{x x}=u(x, t-0.1), \quad x \in(0,1), t \in(0,1], \\
u(x, t)=e^{-x}(1+t), \quad x \in(0,1), t \in[-0.1,0], \\
u(0, t)=1+t, \quad u(1, t)=e^{-1}(1+t), \quad t \in(0,1],
\end{gathered}
$$

where $r(x, t)=2(t+0.95)$. The exact solution of (41) is $u(x, t)=e^{-x}(1+t)$.

Table 1 provides some numerical results of difference scheme (13) solving for (41) with step size $h=\tau=0.01$. Table 2 gives the maximum absolute errors between numerical solutions and exact solutions with different step sizes. From Table 2, we can see that when both the space step 
TABLE 1: Numerical results of (41) when $h=\tau=1 / 100$.

\begin{tabular}{lccc}
\hline$(x, t)$ & Numerical solution & Exact solution & $\left|u\left(x_{i}, t_{k}\right)-u_{i}^{k}\right|$ \\
\hline$(0.5,0.1)$ & 0.667184 & 0.667184 & $2.501 e-007$ \\
$(0.5,0.2)$ & 0.727837 & 0.727837 & $4.324 e-007$ \\
$(0.5,0.3)$ & 0.788490 & 0.788490 & $5.702 e-007$ \\
$(0.5,0.4)$ & 0.849144 & 0.849143 & $6.812 e-007$ \\
$(0.5,0.5)$ & 0.909797 & 0.909796 & $7.753 e-007$ \\
$(0.5,0.6)$ & 0.970450 & 0.970449 & $8.586 e-007$ \\
$(0.5,0.7)$ & 1.031103 & 1.031102 & $9.347 e-007$ \\
$(0.5,0.8)$ & 1.091756 & 1.091755 & $1.006 e-006$ \\
$(0.5,0.9)$ & 1.152409 & 1.152408 & $1.074 e-006$ \\
$(0.5,1.0)$ & 1.213062 & 1.213061 & $1.140 e-006$ \\
\hline
\end{tabular}

TABLE 2: Maximum norm errors of (41) with different step sizes.

\begin{tabular}{lccc}
\hline$h$ & $\tau$ & $E_{\infty}(h, \tau)$ & $E_{\infty}(h, \tau) / E_{\infty}(h / 2, \tau / 2)$ \\
\hline $1 / 10$ & $1 / 10$ & $1.138 e-004$ & $*$ \\
$1 / 20$ & $1 / 20$ & $2.872 e-005$ & 3.962 \\
$1 / 40$ & $1 / 40$ & $7.182 e-006$ & 3.999 \\
$1 / 80$ & $1 / 80$ & $1.796 e-006$ & 4.000 \\
$1 / 160$ & $1 / 160$ & $4.489 e-007$ & 4.000 \\
\hline
\end{tabular}

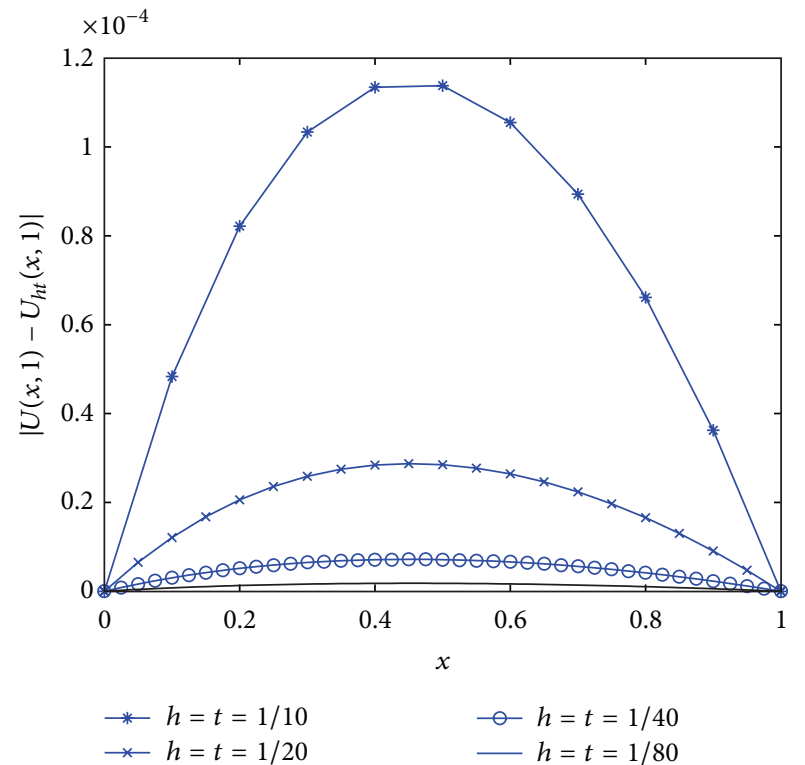

FIGURE 1: Error curves of difference scheme (13) solving for problem (41) with different step sizes, when $t=1$.

size and the time step size are reduced by a factor of $1 / 2$, then the maximum absolute errors are reduced by a factor of approximately $1 / 4$.

Figure 1 provides us with the error curves of numerical solutions for (41) at $t=1$ by using scheme (13). Figures 2 and 3 give the error surface of the numerical solutions with step sizes $h=\tau=1 / 80$ and $h=\tau=1 / 160$, respectively.

Generally speaking, from the results of the tables and the figures provided, we can see that the numerical results are coincident with the theoretical results.

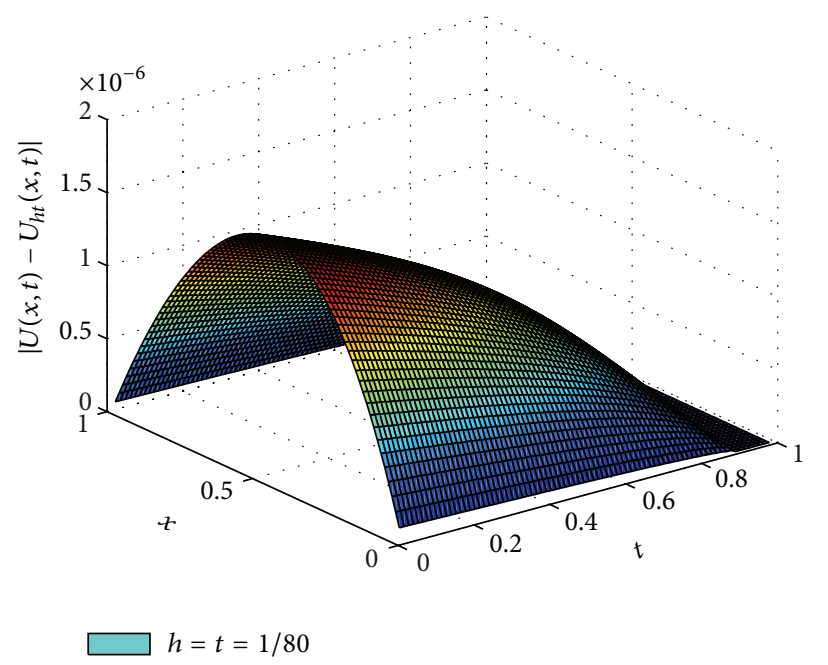

FIGURE 2: Error surface maps of difference scheme (13) solving for problem (41) with step size $h=\tau=1 / 80$.

\section{Conclusion}

In this paper, a type of variable coefficient delay partial differential equations is considered. A linearized Crank-Nicolson scheme is constructed and is proved to be unconditionally stable and convergent. Finally, a numerical test is provided to illustrate the theoretical results.

\section{Conflict of Interests}

The authors declare that there is no conflict of interests regarding the publication of this paper. 


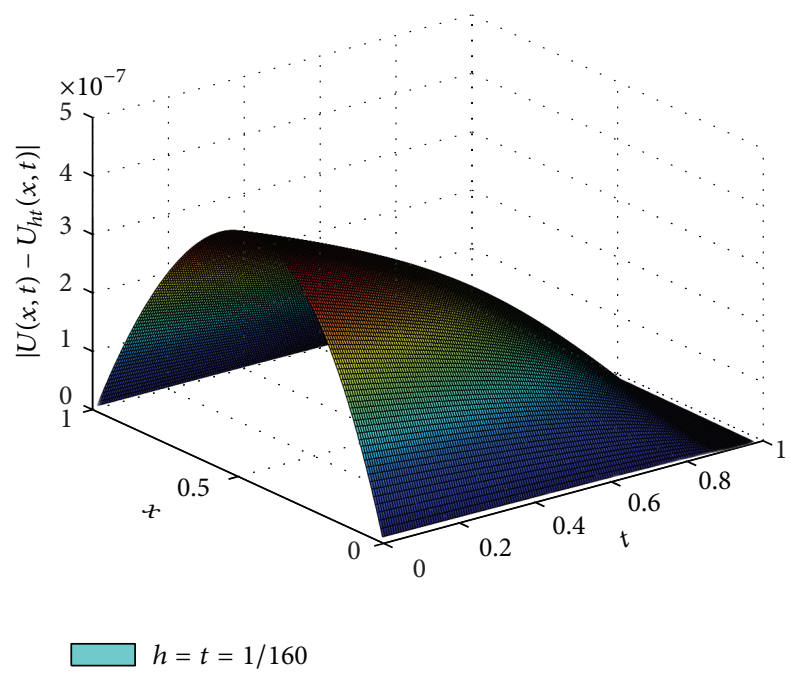

FIGURE 3: Error surface maps of difference scheme (13) solving for problem (41) with step size $h=\tau=1 / 160$.

\section{Acknowledgments}

This work is supported by the Project Sponsored by the Scientific Research Foundation for the Returned Overseas Chinese Scholars, State Education Ministry (no. 2013693), the National Natural Science Foundation of PR China (nos. 71301166, 11301544, 11201487, and 11101184), and the Science Foundation for Young Scientists of Jilin Province (20130522101JH).

\section{References}

[1] D. F. Li and C. J. Zhang, "Nonlinear stability of discontinuous Galerkin methods for delay differential equations," Applied Mathematics Letters, vol. 23, no. 4, pp. 457-461, 2010.

[2] N. V. Azbelev, V. P. Maksimov, and L. F. Rakhmatullina, Introduction to the Theory of Functional Differential Equations, Nauka, Moscow, Russia, 1991.

[3] J. K. Hale, Theory of Functional Differential Equations, Springer, New York, NY, USA, 1993.

[4] O. Diekmann, S. A. van Gils, S. V. Lunel, and H.-O. Walther, Delay Equations: Functional-, Complex-, and Nonlinear Analysis, Springer, New York, NY, USA, 1995.

[5] Z.-B. Zhang and Z.-Z. Sun, "A Crank-Nicolson scheme for a class of delay nonlinear parabolic differential equations," Journal on Numerical Methods and Computer Applications, vol. 31, no. 2, pp. 131-140, 2010.

[6] Z.-Z. Sun and Z.-B. Zhang, "A linearized compact difference scheme for a class of nonlinear delay partial differential equations," Applied Mathematical Modelling, vol. 37, no. 3, pp. 742752, 2013.

[7] Q. F. Zhang and C. J. Zhang, "A new linearized compact multisplitting scheme for the nonlinear convection-reactiondiffusion equations with delay," Communications in Nonlinear Science and Numerical Simulation, vol. 18, no. 12, pp. 3278-3288, 2013.

[8] Z.-Z.Sun, The Numerical Methods for Partial Equations, Science Press, Beijing, China, 2005. (Chinese).
[9] Y. F. Jin, J. X. Jiang, C. M. Hou, and D. H. Guan, "New difference scheme for general delay parabolic equations," Journal of Information and Computational Science, vol. 9, article 18, pp. 55795586, 2012.

[10] C. M. Huang and S. Vandewalle, "An analysis of delay-dependent stability for ordinary and partial differential equations with fixed and distributed delays," SIAM Journal on Scientific Computing, vol. 25, no. 5, pp. 1608-1632, 2004.

[11] C. M. Huang and S. Vandewalle, "Unconditionally stable difference methods for delay partial differential equations," Numerische Mathematik, vol. 122, no. 3, pp. 579-601, 2012. 


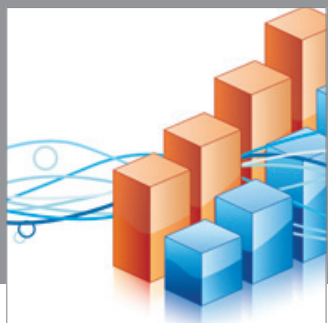

Advances in

Operations Research

mansans

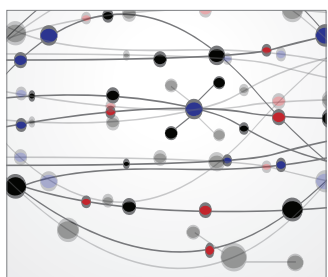

The Scientific World Journal
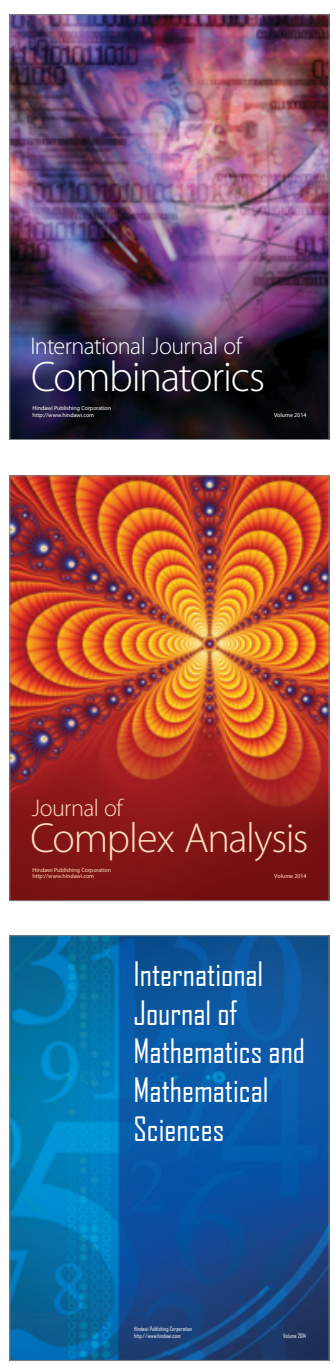
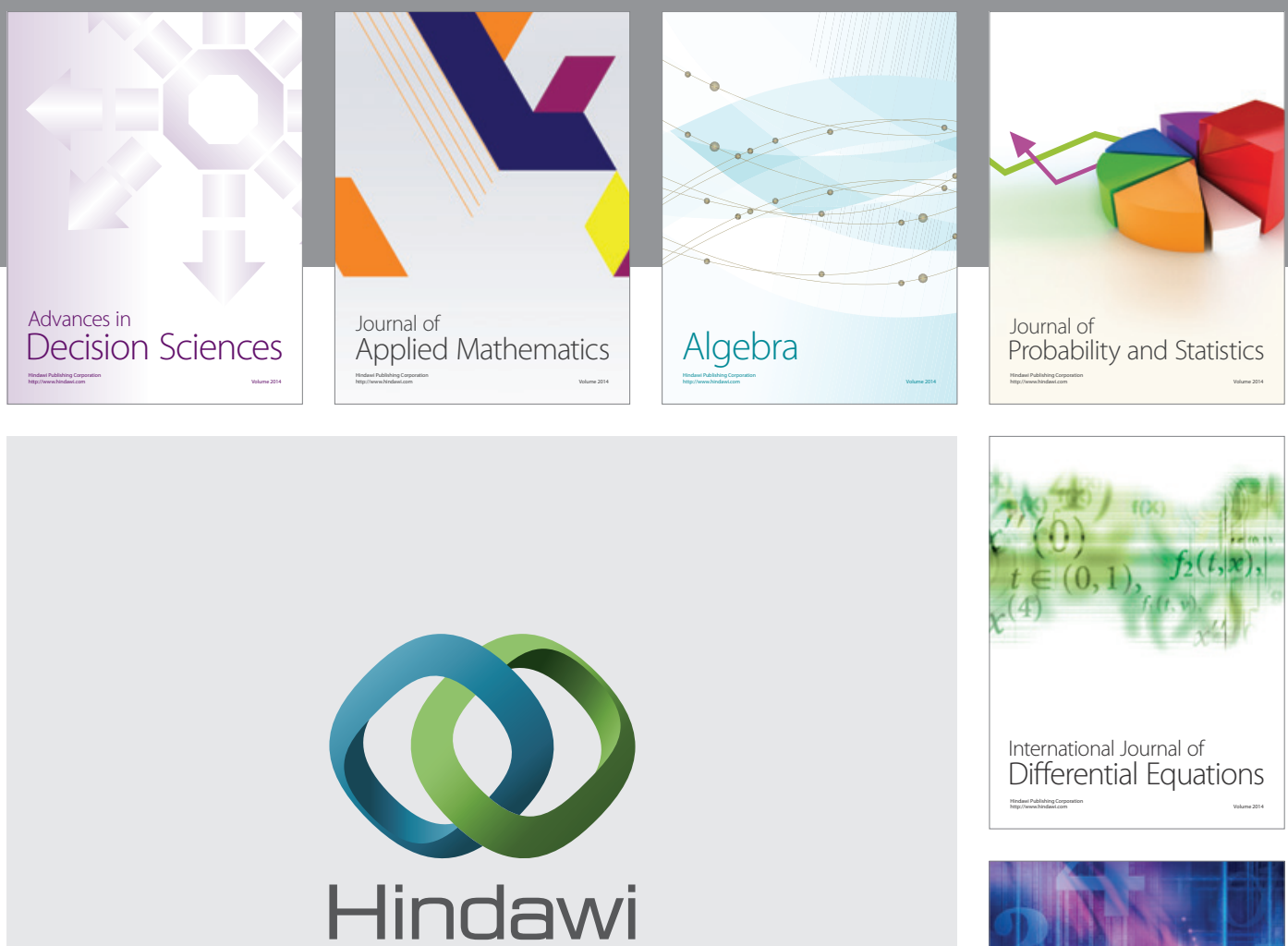

Submit your manuscripts at http://www.hindawi.com
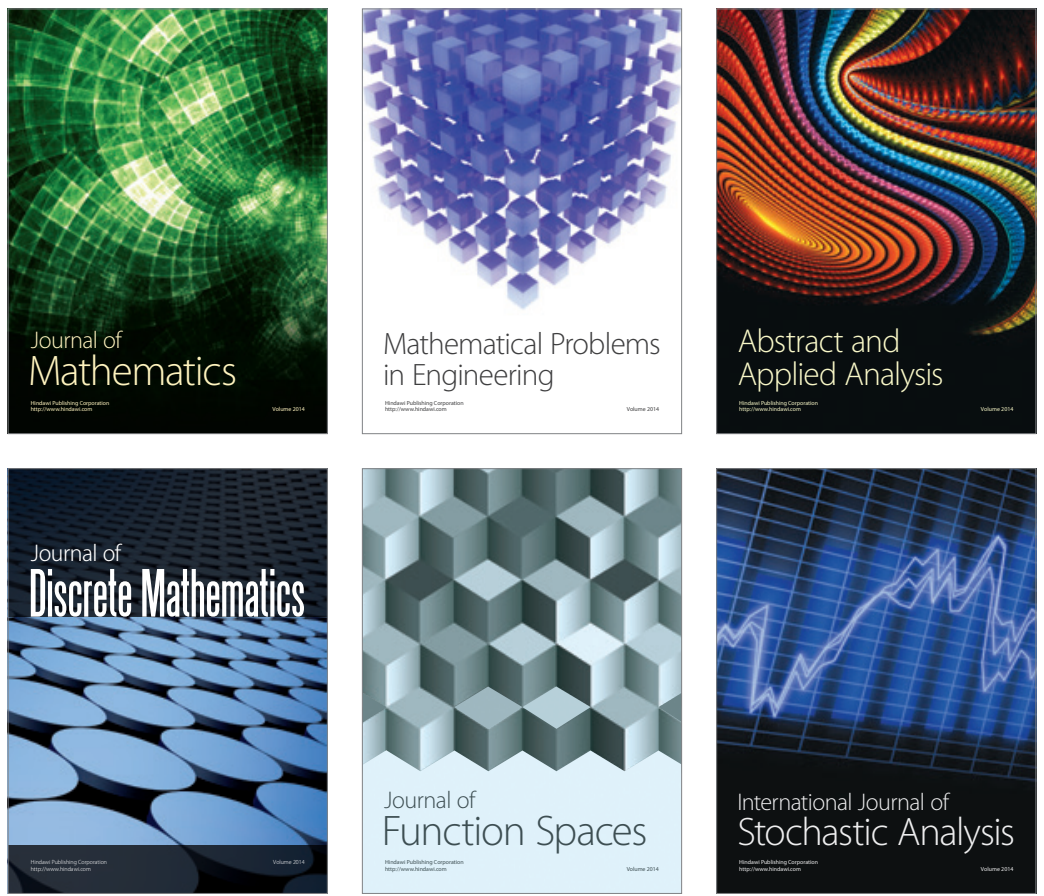

Journal of

Function Spaces

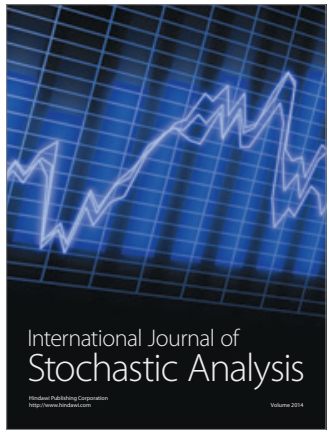

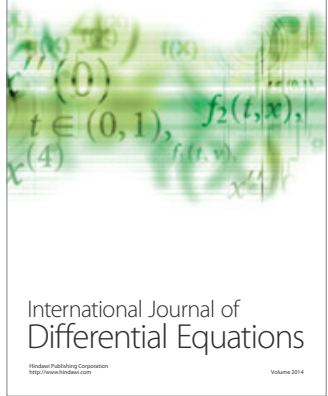
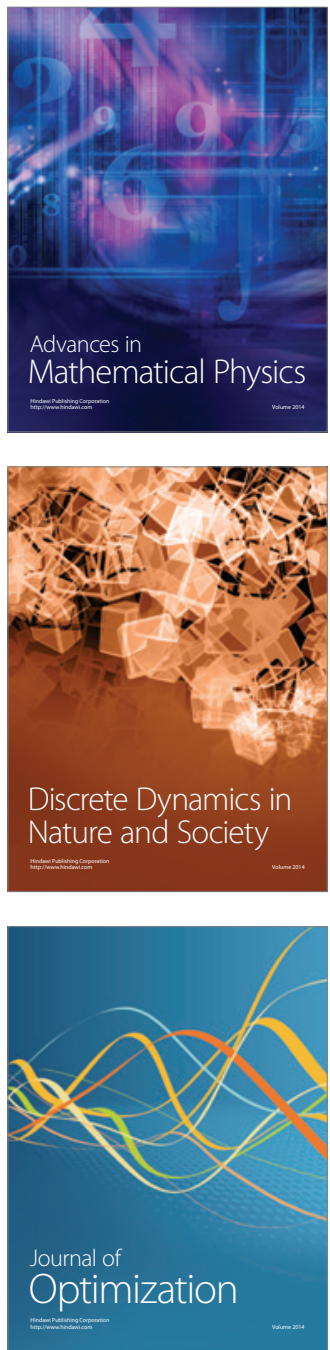